\title{
Effect of Beta Glucan on White Blood Cell Counts and Serum Levels of IL-4 and IL-12 in Women with Breast Cancer Undergoing Chemotherapy: A Randomized Double-Blind Placebo-Controlled Clinical Trial
}

\author{
Alireza Ostadrahimi ${ }^{1}$, Jamal Eivazi Ziaei ${ }^{2}$, Ali Esfahani², Mohammad Asghari \\ Jafarabadi $^{3}$, Aliakbar Movassaghpourakbari², Nazila Farrin ${ }^{4 *}$
}

\begin{abstract}
Background: Breast cancer is the most common female malignancy in the world. Beta glucan can be a hematopoietic and an immune modulator agent in cancer patients. The aim of this trial was to determine the effect of beta glucan on white blood cell counts and serum levels of IL-4 and IL-12 in women with breast cancer undergoing chemotherapy. Materials and Methods: This randomized double-blind placebo-controlled clinical trial was conducted on 30 women with breast carcinoma aged 28-65 years. The eligible participants were randomly assigned to intervention $(n=15)$ or placebo $(n=15)$ groups using a block randomization procedure with matching based on age, course of chemotherapy and menopause status. Patients in the intervention group received two 10-mg capsules of soluble 1-3, 1-6, D-beta glucan daily and the control group receiving placebo during 21 days, the interval between two courses of chemotherapy. White blood cells, neuthrophil, lymphocyte and monocyte counts as well as serum levels of IL-4 and IL-12 were measured at baseline and at the end of the study as primary outcomes of the study. Results: In both groups white blood cell counts decreased after 21 days of the intervention, however in the beta glucan group, WBC was less decreased non significantly than the placebo group. At the end of the study, the change in the serum level of IL-4 in the beta glucan group in comparison with the placebo group was statistically significant $(\mathrm{p}=0.001)$. The serum level of IL-12 in the beta glucan group statistically increased ( $p=0.03$ ) and comparison between two groups at the end of the study was significant after adjusting for baseline values and covariates $(p=0.007)$. Conclusions: The findings suggest that beta glucan can be useful as a complementary or adjuvant therapy and immunomodulary agent in breast cancer patients in combination with cancer therapies, but further studies are needed for confirmation.
\end{abstract}

Keywords: Beta glucan - white blood cell - IL-4 - IL-12 - breast cancer - chemotherapy

Asian Pac J Cancer Prev, 15 (14), 5733-5739

\section{Introduction}

Breast cancer is the most common female malignancy in the world. A remarkable increase in breast cancer incidence has been expressed among Iranian women with significantly vigorous clinical manifestations (Pirouzpanah et al., 2010; Jafari-Koshki et al., 2014; Majeed et al., 2014). It has been showed women aged 40-49 years had the most prevalence of breast cancer in Iran, over $30 \%$ of patients were under 30 years. The incidence rate of breast cancer in women over 30 in Iran is 22 per100,000 (Mousavi et al., 2007). The prevalence rate in this same population is 120 per 100,000 . Glucans are glucose polymers, constitutive a major component of fungal cell wall (Vetvicka, 2011). Glucans are a heterogeneous group of glucose polymers, consisting of a base of $\beta(1,3)$-linked $\beta$-D-glucopyranosyl units with $\beta(1,6)$-linked side chains that the length and distribution of chains are different (Akramiene et al., 2007). Beta glucans have been shown to have pro or anti-inflammatory effects on immune cells (Goodridge et al., 2009; Karaca et al., 2014).These compounds may mediate these activities by stimulating leukocytes and their phagocytic activity, the production of inflammatory mediators, cytokines, and initiation of adaptive immune response (Budak et al., 2008). Studies showed that by using soluble Beta-glucans, a primed state of leukocytes is generated and their response to specific activators is enhanced (Baran et al., 2007). Hematopoietic progenitor and stem cell (HPSC) transplantation has been used widely to treat neoplastic diseases (Cramer et 
al., 2008). Some forms of Beta gleans have anti-tumor properties and stimulate hematopoiesis (Patchen and MacVittie, 1986; Li et al., 2006). Magnani et al (Magnani et al., 2010) study expressed that Carboxymethyl-glucan (CMCM-G) in patients with prostate cancer significantly increased leukocyte count, red blood cell, hematocrit, hemoglobin and platelet counts. A considerable number of cancer patients have been taking complementary medical therapies while receiving their conventional anti-cancer treatments (Chan et al., 2009). In recent years, Beta glucan has been considered in cancer patients for immunomodulation properties (Moore, 2009). Previous Studies suggested that Beta glucans could increase T cell-specific responses by inducing the secrection of INF$\gamma$, TNF- $\alpha, I L-1$, IL-6, IL-8 and IL-12 from macrophages, natural killer cells and neutrophils (Ross et al., 1999; Yan et al., 1999; Yoon et al., 2008).

Beta glucans initiates the immune response by binding to the heterogeneous group of innate immune receptors like lectin receptors, scavenger receptors and Toll- like receptors on the leukocytes (Demir et al., 2007). Dectin-1, is a type II transmembrane protein receptor that binds $\beta-1,3$ and $\beta-1,6$ glucans, can initiate and modulate the innate immune response (Brown et al., 2002; Herre et al., 2004). Brown et al (Brown et al., 2002) recently expressed that dectin-1 is a major Beta Glucan receptor on macrophages. Beta glucan receptors are expressed on monocyte/ macrophages linage and neutrophils. Low expression on splenic dendritic cells surface and specific subgroup of $\mathrm{T}$ cells have been observed (Taylor et al., 2002). Beta glucans containing $\beta-1,3$ and/or $\beta-1,6$ glucan linkages which can interact with the specific receptors of these cells.

Reports in the field of immunity and cancer had demonstrated that there was an imbalance ratio between Th1 and Th2 responses in cancer patients. It have been reported in tumor bearing patients, Th2 immune responses are dominant. Th1 cells increase the cytotoxic T lymphocyte (CTL) response but Th2 cells suppress it, so the cytokine patterns, related to Th1/Th2 balance are main effectors to cancer treatment (Pellegrini et al., 1996; Suzuki et al., 2001; Baran et al., 2007). Regarding to previous studies and positive therapeutic effects of Beta glucan on blood cells and immune responses in cancer patients undergoing chemotherapy, the present study was aimed to investigate the effect of Beta glucan on white blood cell count, their subsets and serum levels of IL-4 and IL-12 in women with breast cancer undergoing chemotherapy.

\section{Materials and Methods}

\section{Study design}

This randomized double-blind placebo-controlled clinical trial with allocation ratio of $(1: 1)$ was carried out from March 2013 to April 2014 in Oncology Ghazi Tabatabayee and Sheikholrais clinics of Tabriz University of Medical Sciences, Tabriz, Iran. The study included women diagnosed as breast cancer patients by Oncologists aged 28-65 years. This clinical trial was approved by the Regional Ethics committee of Tabriz University of
Medical Sciences. Written informed consent was obtained from all patients' at the beginning of the study. Registered No in IRCT is IR201212172017N10.

The sample size was determined based on the data obtained from the Demir et al study (Demir et al., 2007) for Lymphocytes. We considered $\alpha$ value equal to 0.05 and a power of $80 \%$ for calculating the sample size. The sample size was computed as 15 per groups with considering the dropout rate. Inclusion criteria were a definitive diagnosis of breast cancer in stage II and III of disease by oncologists and pathology approving, chemotherapy adjuvant treatment, drink at least $100 \mathrm{ml}$ of water per day, non-allergy for fungi and yeast, adequate bone marrow function, proper functioning of kidneys, The absence of acute medical conditions, women aged 20 years and older, no history of gastrointestinal disease, renal and liver disorders, no consuming prebiotics, probiotics, and antibiotics. Exclusion criteria were patient's unwillingness to participate in the study, pregnancy, lactation, vitamin and mineral users that affected immune system and not receiving blood transfusion.

\section{Patients and variable assessment}

The eligible participants were randomly assigned to intervention or placebo groups by block randomization procedure and subject were matched in each block based on age, course of chemotherapy and menopause status. Finally each group consisted of 15 patients. The follow chart of the study is shown in Figure 1. Patients were recruited between chemotherapy courses of two to four. During the interval two chemotherapy courses (21 days), patients in the intervention group received two 10-mg capsules of soluble 1-3, 1-6, D-beta glucan derived from Saccharomyces cerevisiae (Imuneks, Turkey) daily and the control group receiving placebo in the same shape and size. In order to reduce the dropout rate and to ensure the consumption of the supplements, the subjects received a phone call once per week. Participants were asked to return Beta glucan package in order to assess the supplement consumption of each patient. All the participants were asked not to change their usual dietary intakes, other

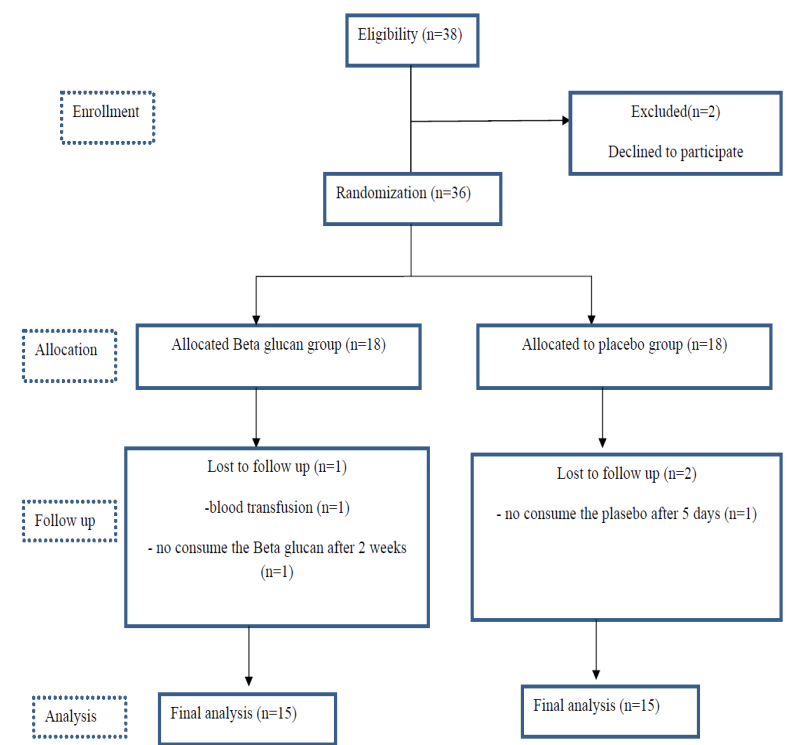

Figure 1. Follow Chart of the Study 
vitamin and minerals supplement consumption, physical activity, medication and traditional medicine as an adjuvant therapy during the study. The patients were asked to inform changing in medicine receiving, blood transfusion during the study. Demographic data including age, course of chemotherapy, type and stage of disease and chemotherapy protocol was obtained by using the questionnaire. Anthropometric indicators including body weight, height, waist circumference (WC) and hip circumference (HC) were measured at beginning and at the end of the study.

Body weight and height were measured using a scale (Seca, Hamburg, Germany) with $0.1 \mathrm{~kg}$ accuracy by wearing light clothing and no shoes and stadiometer with $0.1 \mathrm{~cm}$ accuracy without shoes respectively. Body mass index (BMI) was calculated as body weight in kilograms divided by the square of height in meters $\left(\mathrm{kg} / \mathrm{m}^{2}\right)$. Waist circumference $(\mathrm{WC})(\mathrm{cm})$ was measured at a level of midway between the lower rib margin and iliac crest and hip circumference (HC) at the widest point between the iliac crest and buttock.

Nutrients intake data were collected by using 3-days dietary record at beginning and at the end of the study. Beginning the study all of patients were instructed to how to record and report all of the food they had consumed 3 days by using the food scale at the beginning and at the end of the study. The Nutritionist IV software was used for analyzing 3 days averages of macronutrients and micronutrients intakes.

\section{Blood sampling and biochemical assessment}

Peripheral vein blood samples were drawn at the beginning and at the end of the study. The blood sample was divided into two tubes, one tube contained ethylenediamine-tetraacetic acid in order to measure blood cell count immediately. The other tube was used to separate the blood serum by centrifugation within 30-45 min of collection and serum was stored at $-70^{\circ} \mathrm{C}$ (SANYO, mdf-u33v, Japan, 2010) until they were used for IL-4 and IL-12 assays. CBC was measured by system H1 (Homolog 1) method (Technicon H1system, USA). Serum IL-4 and IL-12 concentrations were measured by enzyme-linked immunosorbent assay (ELISA) using reagent kits of eBioscience (Vienna, Austria) according to the manufacturer's instructions.

\section{Statistical analysis}

The data were analyzed by SPSS 13 (SPSS, Inc. IL, Chicago, USA). The analyses were done by original assigned groups (Intention to treat). Normality of variables distribution was evaluated using Kolmogorov-Smirnov test. Quantitative data were stated as mean \pm (standard deviation (SD)). Non-normal distribution variables were shown as median, (percentile 25 to percentile 75 ). The changes in anthropometric measurements, nutrient intakes, and blood tests of the participants within the beginning and end of the study in each group were compared by using paired sample t-test and non-parametric Wilcoxon Signed Rank -test. The changes in anthropometric measurements, nutrient intakes, and blood tests of the participants between two groups at beginning and end of the study was compared using independent sample t-tests for normal variables and nonparametric Mann-Whitney U test for non-normal variables. Analysis of covariance was used to identify any differences between the two groups after intervention, adjusting for baseline measurements and covariates including energy and BMI differences. Differences with $\mathrm{p}<0.05$ were considered to be statistically significant (AsghariJafarabadi and Mohammadi, 2013).

\section{Results}

Effect of Beta glucan supplementation on body weight and dietary intakes

This randomized, double-blind, placebo-controlled clinical trial was conducted on 30 breast cancer patients according to inclusion and exclusion criteria. Weight, BMI, waist circumference and hip circumference were not shown significant differences between two groups at the beginning and at end of the study. Among study population, in intervention group $26.7 \%, 46.7 \%$ and $26.7 \%$ of patients were on $2^{\text {th }}, 3^{\text {th }}$ and $4^{\text {th }}$ chemotherapy course time respectively. In control group 33.3, 40 and 26.7 percent of patients received $2^{\text {th }}, 3^{\text {th }}$ and $4^{\text {th }}$ chemotherapy course respectively too. In Beta glucan group, women with stage II and stage III of disease were $73.3 \%$ and $26.7 \%$. In control group women with stage II and stage III of disease were $80 \%$ and $20 \%$ respectively. Chemotherapy protocol in two groups included AC (Adriamycin $60 \mathrm{mg} / \mathrm{m}^{2}$ Cyclophosphamide $600 \mathrm{mg} / \mathrm{m}^{2}$ ), CMF (Cyclophosphamide $600 \mathrm{mg} / \mathrm{m}^{2}$, Methotrexate $40 \mathrm{mg} / \mathrm{m}^{2}$, Fluorouracil (5-FU) $600 \mathrm{mg} / \mathrm{m}^{2}$ ) and ECF (Epirubicin 50-100 mg/m², cisplatin $500-600 \mathrm{mg} / \mathrm{m}^{2}$, fluorouracil (5-FU) $500-600 \mathrm{mg} / \mathrm{m}^{2}$ ) and both groups were matched as much as possible. Dietary data and BMI before and after intervention in two groups are presented in Table 1 . There were no statistically significant differences in energy, carbohydrate, protein and total fat values between or within groups at the beginning; however at the end of the study, difference between two groups in energy intake was significant $(\mathrm{p}=0.022)$. There were no statistically significant differences in BMI between or within groups at the beginning and at end of the study. Patients did not express any adverse effects or symptoms with the Beta glucan supplementation.

Effect of beta glucan supplementation on white blood cells, IL-4 and IL-12

At the beginning of the study, there were not significant

Table 1. Dietary Intake and BMI of Patients Before and After the Study

\begin{tabular}{llll}
\hline Variable & Period of study & $\begin{array}{c}\text { Beta glucan Group } \\
(\mathrm{n}=15)\end{array}$ & $\begin{array}{c}\text { Control Group } \\
(\mathrm{n}=15)\end{array}$ \\
\hline Energy intake & Baseline & $1409.7 \pm 254.72$ & $1315.5 \pm 230.98$ \\
$($ kcal $)$ & After intervention & $1488.5 \pm 195.63$ & $1321.6 \pm 179.85^{\text {a }}$ \\
Carbohydrate & Baseline & $194.57 \pm 38.16$ & $190.69 \pm 36.80$ \\
$(\mathrm{~g})$ & After intervention & $215.09 \pm 44.65$ & $197.87 \pm 37.86$ \\
Protein & Baseline & $49.58 \pm 12.08$ & $49.42 \pm 15.53$ \\
$(\mathrm{~g})$ & After intervention & $57.32 \pm 12.11$ & $47.85 \pm 13.25$ \\
Total fat & Baseline & $45.54 \pm 11.23$ & $42.33 \pm 13.39$ \\
$(\mathrm{~g})$ & After intervention & $48.95 \pm 13.82$ & $40.07 \pm 12.25$ \\
BMI & Baseline & $26.29 \pm 4.44$ & $26.02 \pm 3.71$ \\
$\left(\mathrm{Kg} / \mathrm{m}^{2}\right)$ & After intervention & $26.34 \pm 4.40$ & $25.81 \pm 3.65$ \\
\hline adifference between two groups (p=0.022, independent sample t-test $)$
\end{tabular}

Asian Pacific Journal of Cancer Prevention, Vol 15, 2014 
Table 2. Effects of Beta Glucan on White Blood Cell Counts

\begin{tabular}{|c|c|c|c|c|}
\hline & Period of study & Beta glucan Group $(n=15)$ & Control Group $(n=15)$ & Mean Differences $(95 \% \mathrm{CI}, \mathrm{P})$ \\
\hline White Blood Cells & Baseline & $6.19(2.05)$ & $5.61(2.06)$ & $0.57(-0.96-2.11,0.452)^{\mathrm{a}}$ \\
\hline \multirow[t]{3}{*}{ No* $10^{3} / \mu \mathrm{L}[\mathrm{Mean}(\mathrm{SD})]$} & After intervention & $5.99(1.67)$ & $4.91(1.78)$ & $1.08(-0.23-2.40,0.105)^{\mathrm{a}}$ \\
\hline & & & & $0.67(-0.43-1.78,0.225)^{\mathrm{c}}$ \\
\hline & Mean Differences (95\%CI, P) & $0.19(-0.86-1.25,0.697)^{\mathrm{b}}$ & $0.56(-0.34-1.46,0.205)^{\mathrm{b}}$ & \\
\hline Neutrophil & Baseline & $4.01(1.48)$ & $3.40(1.66)$ & No:0.61(-0.57-1.79, 0.299) \\
\hline No* $10^{3} / \mu \mathrm{L}$ & & $61.81(9.95)$ & $60.23(14.73)$ & Percent: $1.58(-7.82-10.98,0.733)^{\mathrm{a}}$ \\
\hline \multirow[t]{6}{*}{ Percent [Mean(SD)] } & After intervention & $3.63(1.51)$ & $3.46(1.21)$ & No:0.17(-0.87-1.21,0.739) $)^{\mathrm{a}}$ \\
\hline & & $61.52(12.69)$ & $65.38(10.85)$ & Percent:-3.86(-12.89-5.16,0.388 $)^{\mathrm{a}}$ \\
\hline & & & & No:- $1.57(-6.16-3.00,0.484)^{\mathrm{c}}$ \\
\hline & & & & Percent:-3.86(-12.79-5.06,0.381 $)^{\mathrm{c}}$ \\
\hline & Mean Differences (95\%CI, P) & No:0.38(-0.47-1.24,0.356) $)^{\mathrm{b}}$ & No:-0.07(-0.84- $0.69,0.843)^{\mathrm{b}}$ & \\
\hline & & Percent:0.29(-5.55-6.14,0.916) ${ }^{\mathrm{b}}$ & Percent:-5.35(-15.78- 5.08, 0.288) & \\
\hline Lymphocyte & Baseline & $1.71(0.87)$ & $1.47(0.58)$ & No:0.24(-0.31-0.79, 0.380$)^{\mathrm{a}}$ \\
\hline No* $10^{3} / \mu \mathrm{L}$ & & $26.56(8.66)$ & $27.93(10.76)$ & Percent: $-1.36(-8.71-5.98,0.706)^{\mathrm{a}}$ \\
\hline \multirow[t]{6}{*}{ Percent [Mean(SD)] } & After intervention & $1.42(0.54)$ & $1.39(0.56)$ & No:0.03(-0.39-0.45,0.877 $)^{\mathrm{a}}$ \\
\hline & & $25.61(9.58)$ & $23.52(8.36)$ & Percent:2.08(-4.78-8.95,0.539) ${ }^{\mathrm{a}}$ \\
\hline & & & & No: $-0.11(-0.35-0.13,0.362)^{c}$ \\
\hline & & & & Percent:2.18(-4.61-8.97,0.514) ${ }^{\mathrm{c}}$ \\
\hline & Mean Differences (CI, p) & No:0.29(0.03-0.54, 0.027) ${ }^{\mathrm{b}}$ & No:0.07(-0.09- $0.25,0.345)^{\mathrm{b}}$ & \\
\hline & & Percent:0.95(-2.63-4.53,0.578) ${ }^{\mathrm{b}}$ & Percent:4.51(-3.58-12.61,0.250) & \\
\hline Monocyte & Baseline & $0.43(0.09)$ & $0.39(0.14)$ & No:0.04(-0.05- $0.13,0.380)^{\mathrm{a}}$ \\
\hline $\mathrm{No}^{*} 10^{3} / \mu \mathrm{L}$ & & $7.34(2.83)$ & $7.55(3.70)$ & Percent:-0.21(-2.68-2.25,0.861 $)^{\mathrm{a}}$ \\
\hline \multirow{6}{*}{ Percent [Mean(SD)] } & After intervention & $0.45(0.15)$ & $0.40(0.17)$ & No:0.05(-0.07-0.17, 0.418$)^{\mathrm{a}}$ \\
\hline & & $8.86(4.78)$ & $7.11(2.74)$ & Percent:1.75(-1.24-4.75,0.241) $)^{\mathrm{a}}$ \\
\hline & & & & No:0.02(-0.09-0.14,0.668) ${ }^{\mathrm{c}}$ \\
\hline & & & & Percent:2.12(-0.70-4.96,0.135) ${ }^{\mathrm{c}}$ \\
\hline & Mean Differences $(95 \% \mathrm{CI}, \mathrm{P})$ & No:-0.02(-0.12-0.06,0.507) $)^{\mathrm{b}}$ & No:-0.01(-0.06- $0.04,0.691)^{\mathrm{b}}$ & \\
\hline & & Percent:-1.52(-3.86- $0.81,0.183)^{\mathrm{b}}$ & Percent:0.69(-1.34-2.72,0.475) ${ }^{\mathrm{b}}$ & \\
\hline
\end{tabular}

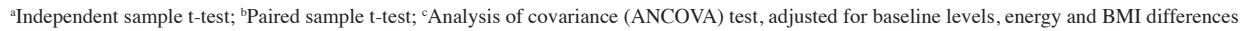

Table 3. Effects of Beta Glucan on Serum Levels of IL-4 and IL-12

\begin{tabular}{|c|c|c|c|}
\hline Period of study & Beta glucan Group $(n=15)$ & Control Group $(n=15)$ & Mean Differences (95\%CI, P) \\
\hline \multicolumn{4}{|c|}{ IL-4(pg/ml) (Median, Percentile 25, Percentile 75) } \\
\hline Baseline & $(1.80,0.40,3.20)$ & $(2.20,1.20,3.50)$ & $0.628^{\mathrm{a}}$ \\
\hline After intervention & $(2.00,1.00,3.00)$ & $(3.80,2.90,4.50)$ & $\begin{array}{c}0.001^{\mathrm{a}} \\
-1.58(-2.70--0.47,0.006)^{\mathrm{e}}\end{array}$ \\
\hline $\mathrm{P}^{\mathrm{b}}$ & 0.824 & 0.092 & \\
\hline \multicolumn{4}{|l|}{ IL-12(pg/ml) Mean(SD) } \\
\hline Baseline & $5.21(3.01)$ & $5.03(0.93)$ & $0.18(-1.68-2.04,0.844)^{\mathrm{c}}$ \\
\hline After intervention & $6.02(3.04)$ & $4.61(0.70)$ & $\begin{array}{l}1.40(-0.53-3.34,0.147)^{\mathrm{c}} \\
1.36(0.40-2.32,0.007)^{\mathrm{e}}\end{array}$ \\
\hline Mean Differences (95\%CI, P) & $-0.81(-1.55--0.07,0.03)^{\mathrm{d}}$ & $0.49(-0.12-1.11,0.108)^{\mathrm{d}}$ & \\
\hline
\end{tabular}

a:Mann-Whitney U test; ${ }^{\mathrm{b}}:$ Wilcoxon Signed Rank -test; ${ }^{\mathrm{i}}$ Independent sample $\mathrm{t}$-tes; ${ }^{\mathrm{A}}$ Paired sample $\mathrm{t}$-test $;{ }^{\mathrm{e}}$ Analysis of covariance (ANCOVA) test, adjusted for baseline levels, energy and BMI differences

differences between two groups in White Blood Cells, neutrophil, lymphocyte and monocyte counts (Table 2). In both groups white blood cells counts decreased after 21 days of chemotherapy; however in Beta glucan group, decrease was less than control group but the comparison between two groups was not significant. Results of covariance analysis for white blood cells showed no significant differences at the end of study, adjusted for baseline levels, energy and BMI differences. At the beginning of the study, there were no significant differences between two groups in monocyte number and percent. At the end of the study monocyte number and percent in Beta glucan group was increased although statistically was not significant by comparison between two groups and after adjusting for baseline values, energy and BMI differences.

The effect of Beta glucan on Serum levels of IL-4 and IL-12 is noted in Table 3. According to table 3 there were no significant differences in serum level of IL-4 and IL-12 at beginning of the study. At the end of the study the change in the serum level of IL-4 in beta glucan group in comparison with the placebo group was statistically significant $(\mathrm{p}=0.001)$. However the serum level of IL-4 changes were not significant within the groups compared with the baseline values. At the end of the study the serum level of IL-12 in Beta glucan group statistically increased $(\mathrm{p}=0.03)$ and in control group decreased in comparison to baseline value although this decrease was not significant. At the end of the study the serum level of IL-12 between two groups was not shown significant differences, although results of analysis of covariance showed significant differences $(p=0.007)$ at the end of study, adjusted for baseline levels, energy and BMI differences.

\section{Discussion}

The present study was aimed to investigate the effect of Beta glucan on white blood cell count, and serum levels of IL-4 and IL-12 in breast cancer patients during the courses 2 th to 4 th of chemotherapy. According to our findings in both groups, white blood cells counts decreased after 21 days; however in Beta glucan group, decrease was less than control group. Serum level of IL-4 in Beta glucan group was decreased in comparison with control group and this reduction statistically was significant. Serum level of 
IL-12 in Beta glucan group statistically increased and in control group decreased none significantly in comparison to baseline value. There are few studies on the effect of Beta glucan supplementation in cancer patients receiving chemotherapy.

Studies showed carboxymethyl Glucan stimulates retioculoendothelial system function and modulates cellular and humoral immunity. In vivo studies showed Beta glucan in the soluble form acts with myeloid growth factors, healing hematopoietic recovery and mobilizing precursor cells in the peripheral blood (Magnani et al., 2010). Pospisil et al. (1991) demonstrated carboxymethyl Glucan increased hemopoietic improvement in sublethally gamma irradiated mice. A series of studies have shown application of Beta glucan and derivatives can increase blood cell counts after cancer treatments (Pospisil et al., 1991; Vetvicka et al., 2007). Demir et al. (2007) suggested that short term oral beta glucan treatment causes to stimulate proliferation and activation of peripheral blood monocytes in vivo with no clinical side effects.

One study (Weitberg, 2008) on patients with advanced malignancy undergoing chemotherapy cleared $\beta-(1,3) /$ $(1,6)$ D-glucan treatment may have beneficial effects on the blood cell counts. Researchers stated B- $(1,3) /$ $(1,6)$ D-glucan is much well-tolerated in patients with advanced malignancies receiving chemotherapy. No adverse effects or toxicities were seen by patients compared to the symptoms at the beginning of the study. Interestingly numerous of patients with malignancy have expressed a sense of well- being when using the $\beta-(1,3) /$ $(1,6)$ D-glucan. It have been reported (Lin et al., 2007) that Maitake beta-glucan (MBG) induce hematopoietic stem cell proliferation and differentiation in colony formation unit response to granulocytes-macrophages in umbilical cord blood cells and it can induce directly granulocyte colony-stimulating factor (G-CSF) in CB CD33+ monocytes. In fact, as mentioned earlier, Beta glucan can stimulate hematopoiesis and leukocytes generation. Although in our study white blood cells count decreased in Beta glucan and control groups whom received chemotherapy but in Beta glucan consumption group this reduction was lower than control group, these results may be related to hematopoiesis properties of Beta glucan. As well as in our study the monocyte number in Beta glucan group increased slightly but this increasing was not significant regardless to other studies.

Our results also showed that Beta glucan supplementation significantly decreased serum level of IL-4 as a Th2 cytokine compared to the control group. Also serum level of IL-12 in Beta glucan group significantly increased in comparison to baseline value. Immune system suppression is observed in most malignancies. In antitumor immunity, T lymphocytes, play a focal regulatory role in cell mediated immunity (Seliger, 2005). New insights in the molecular and cellular mechanisms have cleared that outcome of an immune response toward an evolving breast neoplasm is largely determined by the type of immune responses (DeNardo and Coussens, 2007). It has been shown that balance between $\mathrm{Th} 1 / \mathrm{Th} 2$ controls cytokines production and plays an important role in immuneregulation, including anti-tumor immunity.IL-12, a Th1 related cytokine, shows a strong anti-tumor activity in vivo condition and can modulate $\mathrm{Th} 2$ responses (Nishimura et al., 2000). Studies showed pro-inflammatory cytokines such as IL-2, IL-6 and IL-12 triggers the immune system and they can minimize tumor development or progression. (Foa et al., 1992; Wang et al., 2012). Several mechanisms have been proposed to explain the anti tumor effect of IL12. IL-12 induces pro-inflammatory cytokines synthesis such as TNF- $\alpha$ and IFN- $\beta$ from Th1 cells, induces INF- $\gamma$ production by human T cells and inhibits synthesis of antiinflammatory cytokines such as IL-4, IL-5 and IL-10 by Th2 cells (Li et al., 2009). Th1 and Th2 have antagonistic properties, producing two types of cytokine (Goto et al., 1999). It has been shown Beta glucan is an immune modulator that influences the $\mathrm{T}$ cells, macrophages and natural killer cells functions and increases proliferation of T and B lymphocytes. So, Beta glucan have beneficial effects on inflammatory responses and can be a modulator for anti- inflammatory responses such as interleukin mediators. Some series of studies showed Beta glucan can be useful in cancer condition and cancer patients by immunomodulatory properties (Yoshino et al., 2000; Baran et al., 2007; Wang et al., 2012).

It has been shown (Baran et al., 2007) in vivo application of $\beta$ - 1,3 glucan - WGP from baker's yeast stimulated macrophages to produce IL-12. IL-12 derived from macrophages can induce IFN $\gamma$ production from $\mathrm{T}$ cells, thereby increases Th1 response. The authors declined in vivo usage of Beta- glucan in mammary tumorbearing mice enhanced immunotherapy of cancers. Li et al (Li et al., 2010) expressed orally administered particulate $\beta$-glucan in mice activated dendritic cells that arrested dying tumor cells in vivo, causing to the expansion and activation of antigen-specific CD4 and CD8 T cells. Li et al also cleared tumor burden reduced since Beta glucan treatment significantly increased IFN $-\gamma$ production of tumor-infiltrating $\mathrm{T}$ cells and cytotoxic $\mathrm{T}$ lymphocyte (CTL) responses. So, Beta glucan treated tumors showed DC infiltration with the activated phenotype and significant increasing levels of Th1 cytokines in the tumor microenvironment.

In the study conducted by Yoshino et al. (2000), the authors suggested that in vivo lenthinan consumption have alerted Th1/Th2 balance to Th1 and clearly can cancel Th2-dominant status in patients with digestive cancers. Our findings are consistent with Wang et al. (2012) study on application of lentinan in patients with esophageal carcinoma undergoing treatment for EC. The researchers concluded serum levels of IL-2, IL-6 and IL-12 increased while serum levels of IL-4, IL-5 and IL-10 decreased in lentinan and control groups, however theses changes in experimental group were more than control group.

In fact as mentioned, Beta glucan is an immune modulator that influences the $\mathrm{T}$ cells functions and immune Th1/Th2 balance which controlled with cytokines production by Th1 and Th2. So, Beta glucan plays an important role in immune-regulation such as anti- tumor immunity in cancer patients.

To the best of our knowledge there have been few studies in the effect of Beta glucan on cytokines profiles in breast cancer patients receiving chemotherapy and some 
of our results were in consistent with clinical trials in this field and other cell line and animal studies.

The limitation of this study included a short time of intervention; so long term supplementation with Beta glucan in breast cancer patients should be further investigated in combination chemotherapy courses. The strength of our study is the high acceptance of Beta glucan in breast cancer women undergoing chemotherapy. As well as weekly monitoring of patients by phone call.

In conclusion, the results of this study showed that Beta glucan consumption in breast cancer patients undergoing chemotherapy increased serum level of IL-12 in intervention group and change in serum level of IL-4 in Beta glucan group comparison with placebo group was significant after 21 days. Our results also showed white blood cells counts decreased after 21 days of the intervention, however in Beta glucan group, white blood cells lower decreased in comparison to control group. These findings suggest that Beta glucan usage can be useful as a nutrition adjuvant therapy in combination cancer treatments. Further studies by over the intervention time and larger sample sizes are needed in order to confirm positive effects of Beta glucan supplementation in breast cancer patients' undergoing chemotherapy.

\section{Acknowledgements}

We thank all the patients who participated in this study. The authors are grateful to Hematology and Oncology Research Center of Tabriz University of Medical Sciences for their financial support. This article was written based on a dataset of Ph.D thesis, registered in Tabriz University of Medical Sciences.

\section{References}

Akramiene D, Kondrotas A, Didziapetriene J, Kevelaitis, E (2007). Effects of beta-glucans on the immune system. Medicina, 43, 597-606.

Asharijafarabadi M, Mohammadi S (2013). Statistical series: summarizing and displaying data. J Diabetes Lipid Dis, 12, 83-100.

Baran J, Allendorf DJ, Hong F, Ross GD (2007). Oral beta-glucan adjuvant therapy converts nonprotective $\mathrm{Th} 2$ response to protective Th1 cell-mediated immune response in mammary tumor-bearing mice. Folia Histochem Cytobiol, 45, 107-14.

Brown GD, Taylor PR, Reid DM, et al (2002). Dectin-1 is a major beta-glucan receptor on macrophages. J Exp Med, 196, 407-12.

Budak F, Goral G, Oral HB (2008). Saccharomyces cerevisiae beta-glucan induces interferon-gamma production in human $\mathrm{t}$ cells via IL-12. Turk J Immunol, 13, 21-6.

Chan GC, Chan WK, Sze DM (2009). The effects of beta-glucan on human immune and cancer cells. J Hematol Oncol, $2,25$.

Cramer DE, Wagner S, Li B, et al (2008). Mobilization of hematopoietic progenitor cells by yeast-derived $\beta$-glucan requires activation of matrix metalloproteinase 9. Stem Cells, 26, 1231-40.

Demir G, Klein HO, Mandel-Molinas N, Tuzuner N (2007). Beta glucan induces proliferation and activation of monocytes in peripheral blood of patients with advanced breast cancer. Int Immunopharmacol, 7, 113-6.

DeNardo DG, Coussens LM (2007). Inflammation and breast cancer. Balancing immune response: crosstalk between adaptive and innate immune cells during breast cancer progression. Breast Cancer Res, 9, 212.

Foa R, Guarini A, Gansbacher B (1992). IL2 treatment for cancer: from biology to gene therapy. Br J Cancer, 66, 992-8.

Goodridge HS, Wolf AJ, Underhill DM (2009). Beta-glucan recognition by the innate immune system. Immunol Rev, 230, 38-50.

Goto S, Sato M, Kaneko R, et al (1999). Analysis of Th1 and Th2 cytokine production by peripheral blood mononuclear cells as a parameter of immunological dysfunction in advanced cancer patients. Cancer Immunol Immunother, 48, 435-42.

Herre J, Gordon S, Brown GD (2004). Dectin-1 and its role in the recognition of $\beta$-glucans by macrophages. Molecular immunology, 40, 869-76.

Jafari-Koshki T, Schmid VJ, Mahaki B (2014). Trends of breast cancer incidence in Iran during 2004-2008: A Bayesian space-time model. Asian Pac J Cancer Prev, 15, 1557-61.

Karaca H, Bozkurt O, Ozaslan E, et al (2014). Positive effects of oral beta-glucan on mucositis and leukopenia in colorectal cancer patients receiving adjuvant folfox-4 combination chemotherapy. Asian Pac J Cancer Prev, 15, 3641-4.

Li B, Allendorf DJ, Hansen R, et al (2006). Yeast beta-glucan amplifies phagocyte killing of iC3b-opsonized tumor cells via complement receptor 3-Syk-phosphatidylinositol 3-kinase pathway. J Immunol, 177, 1661-9.

Li B, Cai Y, Qi C, et al (2010). Orally administered particulate beta-glucan modulates tumor-capturing dendritic cells and improves antitumor T-cell responses in cancer. Clin Cancer Res, 16, 5153-64.

Li XH, Ma J, Wu XX, et al (2009). Effect of various combinations of IL2, IL12 and IL15 on function of human peripheral blood derived NK cells. Zhongguo Shi Yan Xue Ye Xue Za Zhi, 17, 918-23.

Lin H, Cheung SW, Nesin M (2007). Enhancement of umbilical cord blood cell hematopoiesis by maitake beta-glucan is mediated by granulocyte colony-stimulating factor production. Clin Vaccine Immunol, 14, 21-7.

Magnani M, Castro-Gomez RH, Aoki MN, et al (2010). Effects of carboxymethyl-glucan from Saccharomyces cerevisiae on the peripheral blood cells of patients with advanced prostate cancer. Exp Ther Med, 1, 859-62.

Majeed W, Aslam B, Javed I, et al (2014). Breast cancer: major risk factors and recent developments in treatment. Asian Pac J Cancer Prev, 15, 3353-8.

Moore MA (2009). Diverse influences of dietary factors on cancer in Asia. Asian Pac J Cancer Prev, 10, 981-6.

Mousavi SM, Montazeri A, Mohagheghi MA, et al (2007). Breast cancer in Iran: an epidemiological review. Breast $J, \mathbf{1 3}, 383-91$.

Nishimura T, Nakui M, Sato M, et al (2000). The critical role of Th1-dominant immunity in tumor immunology. Cancer Chemother Pharmacol, 46, 52-61.

Patchen ML, MacVittie TJ (1986). Hemopoietic effects of intravenous soluble glucan administration. $J$ Immunopharmacol, 8, 407-25.

Pellegrini P, Berghella AM, Del Beato T, et al (1996). Disregulation in TH1 and TH2 subsets of CD4+ T cells in peripheral blood of colorectal cancer patients and involvement in cancer establishment and progression. Cancer Immunol Immunother, 42, 1-8.

Pirouzpanah S, Taleban FA, Atri M, Abadi AR, Mehdipour $P$ (2010). The effect of modifiable potentials on hypermethylation status of retinoic acid receptor-beta2 and estrogen receptor-alpha genes in primary breast cancer. Cancer Causes Control, 21, 2101-11.

Pospísil M, Sandula J, Pipalová I, Hofer M, Viklická S (1991). 
Hemopoiesis stimulating and radioprotective effects of carboxymethylglucan. Physiol Res, 40,377-80.

Ross GD, Vetvicka V, Yan J, Xia Y, Vetvickova J (1999). Therapeutic intervention with complement and beta-glucan in cancer. Immunopharmacology, 42, 61-74.

Seliger B (2005). Strategies of tumor immune evasion. BioDrugs, 19, 347-54.

Suzuki Y, Adachi Y, Ohno N, Yadomae T (2001). Th1/Th2Balancing immunomodulating activity of gel-forming (1-->3)-beta-glucans from fungi. Biol Pharm Bull, 24, 811-9.

Taylor PR, Brown GD, Reid DM, et al (2002). The beta-glucan receptor, dectin-1, is predominantly expressed on the surface of cells of the monocyte/macrophage and neutrophil lineages. J Immunol, 169, 3876-82.

Vetvicka V (2011). Glucan-immunostimulant, adjuvant, potential drug. World J Clin Oncol, 2, 115-9.

Vetvicka V, Dvorak B, Vetvickova J, et al (2007). Orally administered marine (1-->3)-beta-D-glucan Phycarine stimulates both humoral and cellular immunity. Int J Biol Macromol, 40, 291-8.

Wang JL, Bi Z, Zou JW, Gu XM (2012). Combination therapy with lentinan improves outcomes in patients with esophageal carcinoma. Mol Med Rep, 5, 745-8.

Weitberg AB (2008). A phase I/II trial of beta- $(1,3) /(1,6)$ D-glucan in the treatment of patients with advanced malignancies receiving chemotherapy. J Exp Clin Cancer Res, 27, 40.

Yan J, Vetvicka V, Xia Y, et al (1999). Beta-glucan, a "specific" biologic response modifier that uses antibodies to target tumors for cytotoxic recognition by leukocyte complement receptor type 3 (CD11b/CD18). J Immunol, 163, 3045-52.

Yoon TJ, Kim TJ, Lee H, et al (2008). Anti-tumor metastatic activity of beta-glucan purified from mutated Saccharomyces cerevisiae. Int Immunopharmacol, 8, 36-42.

Yoshino S, Tabata T, Hazama S, et al (2000). Immunoregulatory effects of the antitumor polysaccharide lentinan on Th1/ $\mathrm{Th} 2$ balance in patients with digestive cancers. Anticancer Res, 20, 4707-11. 